\section{Internación psiquiátrica involuntaria. Antecedentes, reflexiones y desafíos}

\author{
JUAN ANTONIO BUSTAMANTE DONOSO ${ }^{1, a}$, \\ ALVARO CAVIERES FERNÁNDEZ ${ }^{1,2}$
}

\section{Involuntary psychiatric hospital admissions}

The United Nations Convention on the Rights of Persons with Disabilities Article 12 General Commentary, explicitly states that persons with mental illnesses must always have full exercise of their legal rights in all their aspects. Assistants or support persons must not substitute or have undue influence on the decisions of persons with disabilities, including the expression of their consent. However, the number of involuntary psychiatric hospitalizations appears to be increasing globally and in our country. This article reviews the national and international context of involuntary hospitalizations, including the Chilean legislation and administrative rules, the published evidence about patients' outcomes and clinician attitudes. The authors conclude that the conflict in this issue is not limited to the respect for autonomy and the obligation to provide care. We are now experiencing a new way of caring patients. The complex local, social and cultural realities, require a renewed knowledge and documentation of experiences.

(Rev Med Chile 2018; 146: 511-517)

Key words: Commitment of Mentally III; Involuntary Treatment; Patient Rights; Psychiatry; Psychotic Disorders.

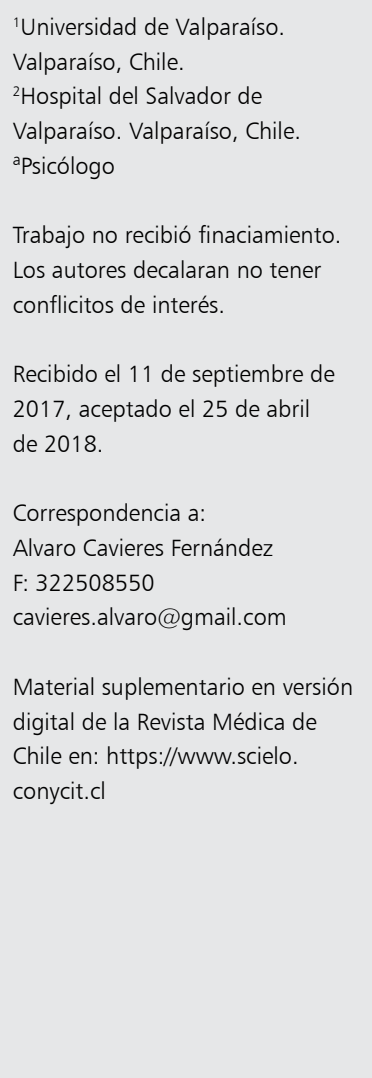

'Universidad de Valparaíso.

Valparaíso, Chile.

${ }^{2}$ Hospital del Salvador de

Valparaíso. Valparaíso, Chile.

apsicólogo

Trabajo no recibió finaciamiento.

Los autores decalaran no tener

2017, aceptado el 25 de abril

Alvaro Cavieres Fernández

F: 322508550

cavieres.alvaro@gmail.com

Material suplementario en versión digital de la Revista Médica de

Chile en: https://www.scielo conycit.cl

\section{Antecedentes regulatorios}

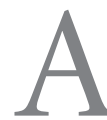

pesar de la dificultad para establecer comparaciones, debido a la implementación de definiciones y políticas diversas, las cifras de hospitalizaciones psiquiátricas involuntarias parecen estar aumentando globalmente. Por contraste, el Comentario General del artículo $12^{1}$, de la Convención de las Naciones Unidas sobre los Derechos de las Personas con Discapacidades ${ }^{2}$, explícitamente señala que, las personas con enfermedades mentales deberán en todo momento, tener ejercicio pleno de sus derechos legales en todos sus aspectos, no pudiendo ser reemplazados por familiares o terceros en la expresión de su consentimiento.

De esta forma, la Convención vuelve a plantear el dilema entre el respeto de la autonomía y libertad de las personas y la obligación de asegurar la máxima protección de la salud y la vida. Esto sin duda, será uno de los aspectos más contro- vertidos del proyecto de ley del reconocimiento y protección de los derechos fundamentales de las personas con enfermedad o discapacidad mental ${ }^{3}$, actualmente en tramitación por el poder legislativo de nuestro país.

En Chile, la normativa legal referente a la internación involuntaria de personas con trastornos psiquiátricos, puede rastrearse hasta enero del año 1927, fecha de publicación del decreto 68, para los servicios de salubridad mental ${ }^{4}$, redactado por el Ministerio de Higiene; Asistencia, Previsión y Trabajo, cuya finalidad era la organización y atención de los servicios de salubridad mental, hospitalización y reclusión de insanos.

Empleando la terminología de la época, el Reglamento denomina a los pacientes como dementes o psicópatas, y los clasifica como, "enfermos alienados y enfermos psicópatas simples". Alienados son enfermos que "deben" ser hospitalizados por mandato de la autoridad a causa de ser antisociales o peligrosos, mientras que, 
"psicópatas simples", serían quienes "pueden" hospitalizarse voluntariamente, sin intervención previa de la autoridad, por ser presuntos dementes, o dementes cuyo estado mental permite considerarlos relativamente "sociales o inofensivos". Un informe médico, debía señalar con claridad, la categoría a la que pertenece cada paciente, y su grado de conciencia de su condición y de oposición al tratamiento.

Los pacientes "alienados" podían ser cuidados en casas particulares, que contaran con la autorización de la Dirección General de Sanidad. Las hospitalizaciones, podían ser voluntarias o de oficio, estas últimas, decretadas por la Justicia, de acuerdo con los códigos Civil y Penal. Por su parte, la Dirección General de Sanidad era responsable de los casos concernientes a los "alienados", ya sea que la internación fuera "pedida a la autoridad competente por cualquiera persona bajo su responsabilidad", o habiendo sido solicitada voluntariamente, "juzgue que deben ser hospitalizados de oficio" ya fuera en una institución pública o privada.

Las hospitalizaciones debían comenzar en un pabellón u hospital psiquiátrico, a menos que una causa de fuerza mayor, obligara a realizarla provisoriamente en otro centro. Después de un mes de su ingreso, la Dirección General de Sanidad determinaría si correspondía el alta, permanencia o traslado del paciente, situación que debía ser revisada periódicamente, y en cualquier momento, a petición del afectado. Se deja constancia que "Solo la autoridad que decretó la hospitalización, podrá autorizar la salida, previo informe médico".

La normativa anterior no fue modificada hasta 1998, cuando se aprobó el decreto supremo $\mathrm{N}^{\circ} 570$ o "Reglamento para la internación de las personas con enfermedades mentales y sobre los establecimientos que la proporcionan" ${ }^{2}$. La enfermedad mental queda definida como "una condición mórbida que sobreviene en una determinada persona, afectando en intensidades variables, el funcionamiento de la mente, el organismo, la personalidad y la interacción social, en forma transitoria o permanente" y se clarifica, específicamente, que se trata de aquellos trastornos incluidos en la décima versión de la Clasificación Internacional de Enfermedades CIE-10, mientras que el diagnóstico de las mismas "corresponderá exclusivamente al médico, el que podrá requerir de la coparticipación de un profesional psicólogo y/o de la colaboración de otros profesionales de la salud con el fin de obtener una evaluación integral del sujeto."

Asimismo, el médico deberá indicar la hospitalización, sólo frente a una de las siguientes situaciones: que el diagnóstico, evaluación clínica o tratamiento, no pueda realizarse en forma ambulatoria, o la presencia de un riesgo de daño físico, psíquico o psicosocial inminente para el paciente o terceros.

Aunque se debe buscar siempre el medio menos privativo de los derechos y libertades de los pacientes, se podrá realizar una hospitalización involuntaria en tres situaciones; para enfrentar una situación de crisis, con una duración máxima de $72 \mathrm{~h}$, posterior a lo cual, de ser necesario, se tramitará con la Autoridad Sanitaria, una internación administrativa, en casos solicitados por la fuerza policial, familiares o miembros de la comunidad, al considerar que la conducta del paciente representa una situación de riesgo o altera el orden o la tranquilidad y finalmente, la internación judicial, dispuesta por algún Tribunal de Justicia.

Durante la hospitalización se deberá requerir el consentimiento del paciente para realizar los tratamientos, a menos que se juzgue que está impedido para hacerlo, en cuyo caso le corresponderá otorgarlo a algún familiar. También se contempla la existencia de un Comité de Ética, al interior de la institución para resolver dilemas clínicos y en el caso de procedimientos irreversibles, la autorización de una Comisión Nacional de Protección de las Personas Afectadas de Enfermedades Mentales.

Las internaciones administrativas serán reevaluadas cada treinta días, debiendo incluir la opinión de más de un médico, e informadas a la autoridad sanitaria que la ordenó, hasta el momento de proponer el alta a esta misma instancia.

Las disposiciones contenidas en el Reglamento 570 del año 1998, son ratificadas y complementadas por la ley 20.584 de 2012, que "regula los derechos y deberes de las personas en relación con acciones vinculadas a su atención en salud"6. Específicamente, el artículo 25, reitera las mismas condiciones para permitir la internación involuntaria, mientras que el artículo 27 , plantea que quienes presenten discapacidad psíquica o intelectual podrán ser tratados involuntariamente, siempre que el diagnóstico esté certificado por un médico psiquiatra, exista un riesgo real e inminente de daño a sí mismo o a terceros, y que suspender o 
no tener tratamiento, significa un empeoramiento de su condición de salud.

En todo caso, el tratamiento no debe extenderse más allá del período estrictamente necesario y debe ser prescrito individualmente, por un médico psiquiatra escogiendo la alternativa terapéutica menos restrictiva posible. Además, se debe tener en cuenta, siempre que sea posible, la opinión de la misma persona, quedando registro en la ficha clínica.

\section{Evidencia de internaciones involuntarias en Chile}

En materia de evidencia, un análisis preliminar, a partir de la solicitud de información AO001T0002370, al Consejo de Transparencia, del 25 de enero de 2017, muestra un aumento en el país, del número de resoluciones de internación administrativa, durante los últimos diez años. Como indicador, podemos ejemplificar los datos de la Región Metropolitana (no se poseen registros previos a estas fechas, acorde a Ordinario A/120 $\mathrm{N}^{\circ} 250$ Subsecretaría de Salud Pública) (Tabla 1). Sin embargo, el porcentaje entre solicitudes y resoluciones varía considerablemente, Valparaíso destaca por la baja cantidad de resoluciones versus solicitudes, además de ser la única región que declara plataforma de gestión en línea.

Un estudio realizado en el sector público de la Región Metropolitana, durante el año 2012, encontró que de un total de 76 solicitudes de internación administrativa, solo $46 \%$ se hicieron

\section{Número de resoluciones de internación administrativa en Chile, según Consejo de Transparencia}

\begin{tabular}{|cc|}
\hline Año & Total resoluciones de internación \\
\hline 2008 & 548 \\
\hline 2009 & 627 \\
\hline 2010 & 579 \\
\hline 2011 & 791 \\
\hline 2012 & 940 \\
\hline 2013 & 894 \\
\hline 2014 & 1.033 \\
\hline 2015 & 744 \\
\hline 2016 & 942 \\
\hline
\end{tabular}

efectivas, correspondiendo en su mayoría a hombres con diagnóstico de esquizofrenia ${ }^{7}$. Estos datos son llamativamente distintos de los reportados en un estudio anterior en un centro privado, donde predominaron las mujeres con diagnóstico de Trastorno Afectivo Bipolar ${ }^{8}$.

\section{Actitudes hacia las internaciones involuntarias}

Las prácticas y decisiones clínicas dan un giro a contar de la década de los 80's. Comenzando en los países de altos y medianos ingresos, se cuestiona el trato a los usuarios como recipientes de beneficencia y paternalismo altruista, implementando políticas , y planes de salud mental, con énfasis en la autonomía de los pacientes y la dignidad humana, fundados en una mirada de Derechos Humanos, aunque con marcadas diferencias entre Estados?

En este contexto, las internaciones involuntarias son controversiales, pues se consideran una seria privación de la libertad individual y la autonomía, pudiendo ser experimentada como gravemente humillante, incluso violenta, reforzando el estigma hacia las personas con trastorno mental y terminar siendo un factor que impide la reinserción, disminuye la adherencia a los tratamientos y empeora el pronóstico ${ }^{10}$.

En los pocos estudios existentes, hay reportes negativos respecto a las consecuencias de las internaciones involuntarias para quienes las experimentan, asociados a sensación de violación de derechos y abuso, restricción de autonomía y limitada participación en el proceso, sensación de no ser cuidado, respetado o escuchado; respuestas emocionales que llevan a sentirse devaluado y estigmatizado $^{11}$. Prácticas como la contención y el aislamiento, son usualmente percibidas como innecesarias, y la experiencia de coerción puede llevar a una sensación internalizada de sí mismo como "loco y malo", resultando en baja autoestima luego del alta.

El estudio de Sibitz, et al $^{11}$, recoge perspectivas en relación a la coerción y la internación involuntaria, que van desde su rechazo completo hasta considerarlo como una ayuda necesaria. Los entrevistados intentan integrar la experiencia en su biografía, como un evento que impacta sobre el resto de su vida, o que motiva al involucramiento en grupos pro derechos de los pacientes, mientras otros prefieren simplemente considerarlo un episodio que es mejor no recordar. 
La gran mayoría de las personas con trastornos psiquiátricos severos viven con sus familias, situación asociada con frecuencia, al estado de Sobrecarga del Cuidador, definido ${ }^{12}$, como "un estado psicológico resultante de la combinación del trabajo físico, la presión social y emocional y las restricciones económicas derivadas del cuidado de los pacientes". En la esquizofrenia, se reportan altos niveles de sobrecarga, en al menos, un tercio de los cuidadores ${ }^{13-15}$, especialmente mujeres ${ }^{16,17}$. Los síntomas psicóticos ${ }^{18,19}$, y deficitarios ${ }^{20}$, una menor compensación y nivel de funcionamiento psicosocial, se asocian con esta situación ${ }^{21}$, así como mayores costos médicos, cesantía y dependencia económica ${ }^{22,23}$.

A pesar de lo anterior, $y$ al hecho de que las familias habitualmente se involucran directamente en la decisión de internar involuntariamente a su pariente enfermo, cuesta encontrar trabajos que aborden el problema desde esta perspectiva. Un estudio cualitativo inglés, reportó que los familiares, junto con expresar alivio, manifestaron sentimientos de culpa después de la internación, además de sentirse poco apoyados para enfrentar la situación de crisis ${ }^{24}$.

La internación psiquiátrica involuntaria ha sido tradicionalmente justificada para prevenir el sufrimiento y dar cuidado apropiado u ofrecer protección hacia el paciente o a terceros. Lo anterior opera bajo cuatro premisas, que son importante revisar y reflexionar, puesto que los equipos trabajan con y a través de ellas: 1) la persona involucrada es considerada incapaz de tomar decisiones racionales sobre el tratamiento; 2) la persona involucrada luego estará agradecida; 3) la evaluación del riesgo para sí mismo y otros es confiable y válida y 4 . las medidas coercitivas son efectivas.

Puestos a revisión, ninguno de los supuestos anteriores tiene apoyo concluyente en la investigación. Es evidente que los pacientes con enfermedad mental pueden tener capacidad de decisión ${ }^{25}$, aunque algunos clínicos pueden sobre estimar su competencia ${ }^{26}$. Por otra parte, el conocimiento empírico sobre los efectos de los cuidados involuntarios es escaso y contradictorio $^{11,27}$. Finalmente, se desconoce si el uso de instrumentos estructurados de evaluación del riesgo mejora la práctica ${ }^{28}$. Todo esto implica que los efectos beneficiosos de la coerción son difíciles de demostrar.

\section{¿Existen alternativas a la internación involuntaria?}

Un metaanálisis publicado recientemente ${ }^{29}$, comparó la efectividad de 4 estrategias, en relación con la disminución del número de hospitalizaciones involuntarias. Las modalidades fueron clasificadas en: mejoramiento de la adherencia al tratamiento; mejoramiento del cuidado habitual; tratamiento ambulatorio involuntario y la declaración de voluntad anticipada. Sólo esta última demostró diferencias estadísticamente significativas a su favor.

Tanto el mejoramiento de la adherencia, como del cuidado habitual, emplean técnicas y estrategias centradas en la provisión del cuidado, incorporando un equipo multidisciplinario y a familiares y cuidadores de los pacientes, en la implementación de programas de psicoeducación, resolución de crisis, tratamiento integral del primer episodio psicótico, etc., en formatos comunitarios y/o de case management $t^{30-32}$.

El Tratamiento Involuntario Ambulatorio $(\mathrm{TAI})^{33-35}$, supone una competencia disminuida del sujeto, para decidir rechazar la medicación y que la suspensión de la misma, implique un riesgo cierto de empeoramiento de su calidad de vida. Esta modalidad, no excluye otro tipo de intervenciones, en especial de tipo psicosocial, ni tampoco las hospitalizaciones forzadas, aunque uno de sus objetivos principales, es disminuir el número de las mismas.

En la práctica, el TAI, está dirigido, especialmente, a personas con esquizofrenia, con problemas reiterados de adherencia. Candidatos con menos probabilidad de beneficiarse, son personas con conductas violentas o de riesgo y quienes evitan activamente los controles de salud.

Los Programas TAI, varían según los países y los servicios que los implementan, pueden consistir en la obligación de asistir a controles, visitas domiciliarias o supervisión de la toma de medicamentos, orales o inyecciones de depósito, e incluir también terapias no farmacológicas. Se recomienda que estas acciones estén incluidas en un plan integral de tratamiento, revisado periódicamente y que se busque algún grado de aceptación por parte del paciente. Si bien, en general, se pretende su mantención por períodos prolongados, su duración es variable, incluyendo intervenciones post-altas, de 3 a 6 meses de duración. 
A pesar de ser visto por muchos, como una alternativa menos restrictiva de tratamiento involuntario, el TAI, no está exento de problemas. Por una parte, requiere desplegar recursos y equipos de profesionales en la comunidad, además de una normativa legal, que considere entre otros aspectos, la autorización para incorporar a un sujeto a la intervención, el empleo de la fuerza pública para hacerla cumplir y el grado de responsabilidad que les corresponde a los médicos por las consecuencias de su indicación. Finalmente, es importante reiterar, que existe poca evidencia, que demuestre su utilidad para disminuir el número de hospitalizaciones involuntarias ${ }^{36}$.

La declaración de Voluntad Anticipada ${ }^{37,38}$ es un formato que prioriza la autonomía del sujeto, quien, durante un período de estabilidad psíquica, define la forma en que desea ser tratado durante una descompensación. Esta declaración puede ser hecha exclusivamente por el paciente o en conjunto con el médico o equipo de salud (Joint Crisis Plan ${ }^{39,40}$, en cuyo caso, también puede participar un facilitador independiente.

Un ejemplo de este tipo de intervención es el Programa CRIMSON ${ }^{41}$, implementado en Inglaterra de manera exploratoria, como un complemento al tratamiento habitual. En dos sesiones, los sujetos participantes expresaron inquietudes y necesidades de cuidado y manifestaron sus preferencias por escrito, después de ser discutidas con el equipo de salud y familiares, en presencia de un facilitador. La declaración quedó incluida en la ficha clínica de los pacientes y pudo ser revisada en un plazo predefinido.

Si bien la evidencia respecto al logro del principal objetivo, la reducción de hospitalizaciones involuntarias es contradictoria, el Programa CRIMSON detectó un aumento en la sensación de empoderamiento de los pacientes, quienes se sintieron más escuchados y respetados. Sin embargo, los autores advierten sobre las dificultades de su implementación, las que incluyen el aumento, para los médicos, de las tareas administrativas, el cambio del modo habitual de relacionarse con los pacientes, y la falta de compromiso de las partes para adherir a los acuerdos ${ }^{42}$.

\section{Reflexiones y desafíos}

El uso de la coerción varía entre y dentro de las jurisdicciones ${ }^{7}$. Su uso depende de la organización de las redes de salud y financiamiento, legislación, características sociodemográficas, patrones de diagnósticos y características de los pacientes.

En un análisis multinivel ${ }^{43}$ de actitudes frente a la coerción, la mayor parte de la varianza pudo ser atribuida a factores individuales del personal sanitario. Feiring y $\mathrm{Ugstad}^{44}$ plantean, en un estudio cualitativo de clínicos de Noruega, que existe una visión que casi análoga la situación requerida para una internación administrativa con la situación de psicosis.

En relación a la voluntariedad, se tiende a asumir que una crisis implica necesariamente, que una persona no es capaz de tomar decisiones racionales, por lo que se evita consultar su parecer. Para los clínicos, el no tomar la opción de internación o tratamiento involuntario, es considerado como inconveniente, por el potencial riesgo posterior. Además la decisión clínica de internar, se basa frecuentemente en la creencia de que la salud será restaurada o significativamente mejorada a través de este proceso.

Existen, sin embargo, discrepancias. Algunos clínicos son críticos y apuntan a que el carácter coercitivo invalida el posible efecto terapéutico. Cuestionando el beneficio de una internación involuntaria, apuntan a una variedad de factores; necesidades, grado de oposición y beneficio esperado de la atención, funcionamiento esperado fuera de la institución, seguimiento profesional, situación de vivienda, red social significativa, personas bajo dependencia, etc.

El conflicto no es sólo entre el respeto por la autonomía y la obligación de provisión de cuidados involuntarios. Estamos frente a un cambio en la visión de los pacientes, y a realidades locales que nos plantean situaciones que se cruzan con complejidades, como el abandono, aislamiento, violencia, situación de calle, precariedad, vulnerabilidad, adicciones, etc. Estas realidades demandan respuestas y soluciones, a través de una coordinación intersectorial, que permita abordar situaciones, donde la salud es uno de los derechos vulnerados, entre otros.

Una vez elaborado un diagnóstico con los diferentes actores involucrados, se debe buscar soluciones creativas, acordes a nuestra realidad. Para ello, se plantea el desafío de generar conocimiento, documentar experiencias, incorporar este interés a las políticas públicas, e identificar oportunidades que permitan encontrar mejores 
soluciones o, al menos limitar, al máximo posible, las internaciones involuntarias.

Los riesgos para los pacientes y sus familiares, nos hablan de un problema de impacto biopsicosocial real, cuya emergencia demanda una respuesta de los servicios de salud, consecuente con un enfoque respetuoso de los derechos de los pacientes. En este sentido, abrir una discusión basada en la evidencia, nos puede dar vías clínicas de acción que permitan innovar y que acompañen las acciones o instancias legales que hoy se encuentra proponiendo el proyecto de Ley de Salud mental.

Material suplementario en versión digital de la Revista Médica de Chile en: https://www.scielo. conycit.cl

\section{Referencias}

1. Observación general sobre el artículo 12: Igual reconocimiento como persona ante la ley Convención Internacional sobre los Derechos de las Personas con Discapacidad. Comité sobre los Derechos de las Personas con Discapacidad. ONU. www.ohchr.org/Documents/ HRBodies/CRPD/GC/DGCArticle12_sp.doc (Consultado el 5 de mayo de 2017).

2. Convención Internacional sobre los Derechos de las Personas con Discapacidad. Naciones Unidas Enable. http://www.un.org/spanish/disabilities/default.asp?navid=12\&pid=497 (Consultado el 6 de marzo de 2009).

3. Proyecto de Ley Establece normas de reconocimiento y protección de los derechos fundamentales de las personas con enfermedad o discapacidad mental https:// www.camara.cl/sala/verComunicacion.aspx?comui$\mathrm{d}=23026 \&$ formato $=$ pdf (Consultado el 5 de mayo de 2017).

4. Decreto 68 Reglamento para los Servicios de Salubridad Mental Santiago de Chile 10 de marzo, 1927 http://bcn. $\mathrm{cl} / 251 \mathrm{bb}$.

5. Decreto 570 Reglamento para la internación de las personas con enfermedades mentales y sobre los establecimientos que la proporcionan Santiago de Chile 14 de julio, $2000 \mathrm{http} / / / \mathrm{bcn} . c \mathrm{l} / 1 \mathrm{v} 4 \mathrm{v} 0$.

6. Ley 20.584 Regula los Derechos y Deberes que tienen las Personas en relación con Acciones Vinculadas a su Atención en Salud. Santiago de Chile 24 de abril, 2012 http://bcn.cl/1uw7l.

7. Cruz C, Martínez $\mathrm{C}_{\check{\iota}}$ Por qué pacientes con internación administrativa no se están hospitalizando ? Psiquiatría y Salud Mental 2016; 33: 16-8.
8. Santander J, Huerta D, Aceitubo D, Fuentes X. Descripción clínica y socio-demográfica de pacientes psiquiátricos internados involuntariamente bajo el régimen de hospitalización administrativa en Chile. Rev Chil Neuro-Psiquiat 2011; 49 (2): 157-64.

9. Steinert $\mathrm{T}$, Lepping $\mathrm{P}, \mathrm{Bernhardsgrütter} \mathrm{R,} \mathrm{Conca} \mathrm{A,}$ Hatling T, Janssen W, et al. Incidence of seclusion and restraint in psychiatric hospitals: a literature review and survey of international trends. Soc Psychiatry Psychiatr Epidemiol 2010; 45: 889-97.

10. Corrigan, P, Rao D. On the Self-Stigma of Mental Illness: Stages, Disclosure, and Strategies for Change. Canadian Journal of Psychiatry 57 (8): 464-9.

11. Sibitz I, Scheutz A, Lakeman R, Schrank B, Schaffer M, Amering M Impact of coercive measures on life stories: qualitative study. BJP 2011; 199 (3): 239-44.

12. Dillehay R, Sandys M. Caregivers for Alzheimer's patients: what we are learning from research. Int J Aging Hum Dev 1990; 30: 263-85.

13. Maurin J, Boyd C. Burden of mental illness on the family: a critical review. Arch Psychiatr Nurs 1990; 4: 99-107.

14. Awad A, Voruganti L. The burden of schizophrenia on caregivers: a review. Pharmacoeconomics 2008; 26: 14962.

15. Magliano L, Fadden G, Madianos M, de Almeida JM, Held T, Guarneri M, et al. Burden on the families of patients with schizophrenia: results of the BIOMED I study. Soc Psychiatry Psychiatr Epidemiol 1998; 33: 405-12.

16. Caqueo A, Gutiérrez J, Kavanagh D. Burden of care in families of patients with schizophrenia. Quality of Life Research 2006; 15: 719-24.

17. Schmid R, Spiessl H, Cording C. Between responsibility and delimitation: emotional distress of caregivers. Psychiatr Prax 2005; 32: 272-80.

18. Woltraus J, Dingemans P. A Caregiver burden in recent onset schizophrenia and spectrum disorders. The influence of symptoms and personality traits J Nerv Ment Dis 2002; 190: 241-7.

19. Magliano L, Fiorillo A, Malangone C, Aletti A, Belotti G, Bevilacqua P, et al. Family burden in schizophrenia: effects of socio-environmental and clinical variables and family intervention. Epidemiol Psichiatr Soc 1998; 7: 178-87.

20. Dyck D, Short R, Vitaliano PP. Predictors of burden and infectious illness in schizophrenia caregivers. Psychosom Med 1999; 61: 411-9.

21. Parabiaghi A, Lasalvia A, Bonetto C, Cristofalo D, Marrella G, Tansella M, et al. Predictors of changes in caregiving burden in people with schizophrenia: a 3-year 
follow-up study in a community mental health service. Acta Psychiatr Scand Suppl 2007; 66-76.

22. Fadden G, Kuipers L. The burden of care: The impact of functional psychiatric illness on the patient's family. BrJPsychiatry 1987; 150: 285-92.

23. Hou S, Ke C, Su Y, Lung FW, Huang C. Exploring the burden of the primary family caregivers of schizophrenia patients in Taiwan. Psychiatry Clin Neurosci 2008; 62: 508-14.

24. Jankovic J, Yeeles K, Katsakou C, Amos T, Morriss R, Rose D, et al. Family caregivers' experiences of involuntary psychiatric hospital admissions of their relatives--a qualitative study. PLoS One 2011; 6 (10): e25425. doi:10.1371/journal.pone.0025425.

25. Owen G, Szmukler G, Richardson G, Davis S, Raymond V, Freyenhagen F, et al. Decision-making capacity for treatment in psychiatric and medical in-patients: cross-sectional, comparative study. BJP 2013, 203: 4617.

26. Lepping P. Overestimating patients' capacity. BJP 2011; 199: 355-6.

27. Prinsen EJD, van Delden JJM. Can we justify eliminating coercive measures in psychiatry? J Med Ethics 2009; 35: 69-73.

28. Singh JP, Fazel S, Gueorguieva R, Buchanan A. Rates of violence in patients classified as high risk by structured risk assessment instruments. BJP 2014; 204: 180-7.

29. de Jong $M H$, Kamperman AM, Oorschot M, Priebe $S$, Bramer W, van de Sande R, et al. Interventions to Reduce Compulsory Psychiatric AdmissionsA Systematic Review and Meta-analysis. JAMA Psychiatry 2016; 73 (7): 657-64.

30. Staring A, Van der Gaag M, Koopmans G, Selten J, Van Beveren J, Hengeveld M, et al. Treatment adherence therapy in people with psychotic disorders: randomised controlled trial. Br J Psychiatry 2010; 197 (6): 448-55.

31. Lay B, Drack T, Bleiker M, Lengler S, Blank C, Rössler W. Preventing Compulsory Admission to Psychiatric Inpatient Care: Perceived Coercion, Empowerment, and Self-Reported Mental Health Functioning after 12 Months of Preventive Monitoring. Front Psychiatry 2015; 6: 161.

32. Dieterich M, Irving CB, Bergman H, Khokhar MA, Park $\mathrm{B}$, Marshall M. Intensive case management for severe mental illness. Cochrane Database Syst Rev 2017; 1: CD007906.

33. O'Reilly R, Dawson J, Burns T. Best practices: Best practices in the use of involuntary outpatient treatment.
Psychiatr Serv 2012; 63 (5): 421-3.

34. Cañete-Nicolás C, Hernández-Viadel M, Bellido-Rodríguez C, Lera-Calatayud G, Asensio-Pascual P, Pérez-Prieto JF, et al. Involuntary outpatient treatment (iot) for severe mental patients: current situation in Spain. Actas Esp Psiquiatr 2012; 40 (1): 27-33.

35. Reitan T. Commitment without confinement. Outpatient compulsory care for substance abuse, and severe mental disorder in Sweden. Int J Law Psychiatry 2016; 45: 60-9.

36. Kisely SR, Campbell LA, O'Reilly R. Compulsory community and involuntary outpatient treatment for people with severe mental disorders. Cochrane Database Syst Rev 2017; 3: CD004408.

37. Henderson C, Swanson JW, Szmukler G, Thornicroft G, Zinkler M. A typology of advance statements in mental health care. Psychiatr Serv 2008; 59: 63-71.

38. Srebnik D, Brodoff L. Implementing psychiatric advance directives: service provider issues and answers. J Behav Health Serv Res 2003; 30: 253-68.

39. Farrelly S, Brown G, Rose D, Doherty E, Henderson R, Birchwood M, et al. What service users with psychotic disorders want in a mental health crisis or relapse: thematic analysis of joint crisis plans. Soc Psychiatry Psychiatr Epidemiol 2014; 49 (10): 1609-17.

40. Farrelly S, Lester H, Rose D, Birchwood M, Marshall $\mathrm{M}$, Waheed $\mathrm{W}$, et al. Barriers to shared decision making in mental health care: qualitative study of the Joint Crisis Plan for psychosis. Health Expect 2016; 19 (2): 448-58.

41. Thornicroft G, Farrelly S, Birchwood M, Marshall M, Szmukler G, Waheed W, et al. CRIMSON [CRisis plan IMpact: Subjective and Objective coercion and eNgagement] protocol: a randomised controlled trial of joint crisis plans to reduce compulsory treatment of people with psychosis. Trials 2010; 11: 102.

42. Thornicroft G, Farrelly S, Szmukler G, Birchwood M, Waheed W, Flach C, et al. Clinical outcomes of Joint Crisis Plans to reduce compulsory treatment for people with psychosis: a randomised controlled trial. Lancet 2013; 381 (9878): 1634-41.

43. Husum T, Bjørngaard J, Finset A, Ruud T. Staff attitudes and thoughts about the use of coercion in acute psychiatric wards. Soc Psychiatry Psychiatr Epidemiol 2011; 46: 893-901.

44. Feiring E, Ugstad K. Interpretations of legal criteria for involuntary psychiatric admission: a qualitative analysis. BMC Health Serv Res 2014; 14: 500. 